Discussion Paper No. 1010

\title{
AN EVOLUTIONARY ANALYSIS OF THE ASSIGNMENT OF PROPERTY RIGHTS
}

Atsushi Tsuneki

September 2017

The Institute of Social and Economic Research Osaka University

6-1 Mihogaoka, Ibaraki, Osaka 567-0047, Japan 
An Evolutionary Analysis of the Assignment of Property Rights*

Abstract: We develop an evolutionary game model to reveal the theoretical basis for the assignment of property right, where both plaintiff and defendant argue for their rights by claiming their reliance investment. We allow for the possibility that the value of the total product depend not only on the investment conferred by the owner but also on the reliance investment provided by the trespasser. The resulting evolutionary stable set of preferences shows that the endowment effect hardwired to the owners and trespassers depends on the difference of productivities among both parties and the density of owners within the population.

Keywords: Evolutionary Game, Property Right, Reliance Investment, Endowment Effect.

JEL Classification Code:C73, K11

\footnotetext{
* Institute of Social and Economic Research, Osaka University. The author thanks Mukesh Eswaran and Hugh Neary for valuable suggestions on the idea of this paper. Remaining errors are the responsibility of the author. This research is supported by JSPS Grant-in-Aid for Scientific Research for Challenging Exploratory Research (No. 26590046).
} 


\section{Introduction}

As pointed out by North (1990), the basic premise of the institutionalist school of economics is the critical role of the system of property rights in the process of economic development. ${ }^{1}$ Because the delineation and protection of the property right are roles of law, this suggests an inalienable relationship between law and economics in the study of social science.

Traditional legal studies and economic analysis of law have taken different paths on the issue. The traditional school of law emphasizes the role of moral principles, such as first possession or reliance reflected in customs and precedents, in the resolution of disputes over property rights, thus promoting procedural fairness and distributive justice among conflicting parties. Unfortunately, traditional analyses lack the theoretical basis of legal principles and, hence, legal rules and principles are sometimes applied by courts in unpredictable ways.

Coase (1960), who provided the basis of law and economics emphasized the efficiency aspect of the assignment of legal rights. His celebrated Coase Theorem shows that the clear and explicit determination of legal rights and private bargaining among private parties can resolve the externality problem, without government intervention, if no transaction costs exist. Furthermore, he emphasizes the reciprocal nature of the externality problem, and shows that the efficient outcome is attained by private bargaining, without depending on the distribution of legal rights assigned to each party. Therefore, for Coase, the assignment of legal rights was a problem of enforcing the law in order to minimize aggregate transaction costs. Coase's idea was succeeded by the classic economic analysis of property law by Posner (1986), who argued that property law should be structured to minimize the transaction costs of private bargaining and the market exchange of properties. ${ }^{2}$

The recent contribution of Eswaran and Neary (2014) proposed an evolutionary approach to reveal the theoretical basis that supports the institution of property rights. According to their analysis, the legal system of property rights is preceded by the evolutionarily developed human psyche, which is attributed either to John Locke's labor theory or to the principle of first possession. These two reasons are quite often adopted within legal discourse to stipulate the existence and assignment of property rights in order to resolve specific disputes. As such, they provide the evolutionary basis for the legal institution of property rights, and its associated legal doctrines, as a sense of

1 See Besley and Ghatak (2010) for a more recent survey on this subject.

2 See also Cooter (1982), who points out the other efficiency aspect of property law: minimizing the cost of the failure of private bargaining over natural rights. This aspect is attributed originally to Hobbes (1651/1973). 
justice, hardwired by the evolutionary process of natural selection.

The chief goal of Eswaran and Neary (2014) was to show that the preference intensities of the original owner and an interloper with regard to a property become different when the former has a production opportunity to enhance the value of the property, or has an incumbency advantage over the latter in terms of securing the fruits from the property. In particular, their study showed that the owner develops a stronger preference intensity on his property than that of the interloper within the evolutionary process when only the owner has an opportunity to enhance the value of the property using his labor input. This is true even if they are ex ante identical with respect to their preference and ability to keep the fruits from the property. This proposition provided a rigorous evolutionary foundation to Locke's labor theory of value (Locke (1689/1988)).

However, when the legal right to a property is contested among people, it is often the case that both the plaintiff and the defendant argue their rights by claiming their reliance investment in the property. For example, when the ownership of land is contested between the legal owner and a de facto owner, both parties often lay claim to their ownership by asserting their contributions to the development and protection of the land.

The purpose of this study is to extend the propositions of Eswaran and Neary (2014) to the above-mentioned case. We start with a model where individuals are identical ex ante, except that some individuals, who we call owners, naturally have their own property (sometimes referred to as land). The remaining individuals are called trespassers, who survive by using some fixed resource with which they are naturally endowed. Owners meet with trespassers with a fixed probability, which depends on the density of owners within the total population, as determined by nature. Once they meet, a struggle for the distribution of the product inevitably occurs, owing to the trespasser's demand to consume the product from the land.

Our model deviates from that of Eswaran and Neary (2014) in that we allow for the possibility that the value of the total product depends not only on the labor investment conferred by the owner, but also on the reliance investment provided by the trespasser to protect and enhance the value of the land.

Consider the case where the owner is a farmer who cultivates corn for his survival. The amount of corn produced depends on the labor of the farmer. However, it also depends on the level of effort supplied by the trespasser when he met with the owner of the property. In order to provide an appropriate level of care to protect and improve the land, the trespasser needs a reliance investment ex ante, as in the case of the owner, who chooses the level of labor to apply to his land. The resulting evolutionary stable set 
of preferences differs from that of Eswaran and Neary (2014) in that the relative endowment effect hardwired to the owner becomes smaller than it does in their case. Furthermore, in some cases, trespassers have more intense preferences for the product generated from the property than the owners do.

The rest of this paper proceeds as follows. Section 2 presents our model, and section 3 presents our principal results, along with intuitive explanations of the results. Then, section 4 discusses the implications of our results for law and economics. Lastly, section 5 concludes the paper.

\section{Model}

We consider an evolutionary model where people live for one period. Their genes are inherited by their offspring, and the biological cycle repeats itself. The natural environment offers a random share $\theta$ of the total population of each generation the possibility of owning some resource, say land, for their survival. These people, who we call owners, invest effort in the resource (e.g., by growing corn on the land). The remaining people (the $1-\theta$ share of the total population) are not originally endowed with land and, thus, they survive using some outside option, such as collecting fruit in a forest. When $\theta \geq \frac{1}{2}$, any trespasser must meet with one owner on his land. In contrast, some trespassers need not meet with an owner when $\theta<\frac{1}{2}$. We assume that any trespasser who meets with an owner must lose his original outside option, and must share the land with the owner and grow corn to survive. This reflects the inevitable nature of the externality we envision in our model. In both cases, once the owner and trespasser meet, there begins a contest over the share of the corn each should receive in order to survive.

We assume that the production function of farming corn on the land is as follows. When the landowner does not meet with a trespasser, he follows a production process represented by the usual production function,

$$
q(K)=A K^{a}, 0 \leq a \leq 1,
$$

where $K$ denotes the level of labor conferred by the owner on his land to produce corn.

In contrast, when the landowner meets with a trespasser, we assume that the aggregate production level of the corn produced by the two people is given by the following production function:

$$
q(K, L)=A K^{a} L^{b}, 0 \leq a, b \leq 1, a+b \leq 1,
$$

where $\mathrm{K}$ and $\mathrm{L}$ denote the input levels of the owner and trespasser, respectively, to produce corn $q$. It may appear to be more natural to assume that the level of production depends on the aggregate level of input $K+L$, as long as the type and quality of the 
effort of the two parties are homogeneous and substitutable. Instead, we assume that there exists some complementarity between $K$ and $L$ by assuming heterogeneous aspects of the inputs used to produce the corn, which are necessary for both parties to protect the land from harm during the production process. If the usage of land is not appropriate (e.g., using incorrect machines for production, or supplying toxic chemicals), the land is devastated and the corn production level becomes very low, irrespective of the amount of labor used. To protect the land from such harm, both parties must put additional effort into protecting the land. In particular, if either side supplies a negligible input as a protective investment, the level of production approaches zero, irrespective of the labor input by the other party. Our assumption on production reflects the cooperative and heterogeneous aspects of the productive inputs supplied by the two parties that become necessary to protect the common resource from fatal deterioration.

Our model consists of three stages. In Stage 1, nature chooses the preference parameters $v$ and $w$ of the owner and trespasser, respectively, as an evolutionary process. Note that we sometimes refer to the owner as Player 1, and the trespasser as Player 2. Then, in Stage 2, Player 1 and Player 2 choose the levels of prior effort $K$ and $L$, respectively, required to increase the level of production, given the preference parameters $v$ and $w$. Finally, in Stage 3, both players contest the distribution of the corn produced in Stage 2. Because we solve the model in a backward manner, we begin by analyzing Stage 3 .

\subsection{Distribution Contest}

By Stage 3, the levels of productive effort, $K$ and $L$, and the output $q$ are already fixed. With natural probability, a distribution contest occurs between the two players to divide the fixed aggregate production $q$. We denote the levels of effort required by Players 1 and 2 to receive more of the product for consumption as $e$ and $f$, respectively. We assume that the preference parameters $v$ and $w$, which are predetermined in Stage 1 , are observable to each player in the distribution contest in Stage 3. Our model closely follows Eswaran and Neary (2014), as does the justification for this assumption. ${ }^{3}$

We further assume that the effectiveness of the effort for distribution is equal for both players in order to reflect the equality of people in the state of nature. Therefore, the shares of corn produced by Players 1 and 2 ( $s$ and $t$, respectively) are determined as follows:

$$
\mathrm{s}=\frac{e}{e+f}, t=1-s=\frac{f}{e+f}
$$

3 See Eswaran and Neary (2014:220-221). 
In Stage 3, both players maximize their utility functions:

$$
\begin{gathered}
\max _{e} v \ln (s q)-(e+K) \\
\max _{f} w \ln (t q)-(f+L),
\end{gathered}
$$

where $\ln (c)$ is the biological level of the fitness of the consumption, common to all generations and types of players. In the process of choice in one generation, people deviate from the natural fitness level of consumption $\ln (c)$, depending on the parameters that reflect the intensity of the players' preferences, $v \ln (c)$ and $w \ln (c)$. These parameters are the result of an evolutionary process, which we analyze in the next section.

We can explicitly solve for the Nash equilibrium effort levels for both players, as follows.

$$
\begin{aligned}
& e^{*}(v, w)=\frac{v \sqrt{w}}{\sqrt{v}+\sqrt{w}}, f^{*}(v, w)=\frac{\sqrt{v} w}{\sqrt{v}+\sqrt{w}} \\
& s^{*}(v, w)=\frac{\sqrt{v}}{\sqrt{v}+\sqrt{w}}, t^{*}(v, w)=1-s^{*}(v, w) .4
\end{aligned}
$$

It is easy to check that $\frac{\partial e^{*}}{\partial v}, \frac{\partial f^{*}}{\partial w}, \frac{\partial s^{*}}{\partial v}$, and $\frac{\partial t^{*}}{\partial w}>0$, which means that the players' equilibrium effort levels and their shares of the output increase as their preference intensities increase in the evolutionary process.

\subsection{Stage 2: Choice of Production Inputs}

Denote $\mu=\min \left[1, \frac{1-\theta}{\theta}\right]$ and $\varphi=\min \left[1, \frac{\theta}{1-\theta}\right]$, where $\mu$ is the probalitity that the owner meets with a trespasser, and $\varphi$ is the probability that the trespasser meets with an owner.

The production decisions of the owner and trespasser are formalized respectively as follows:

$$
\max _{K} \mu\left\{v \ln \left(s^{*} A K^{a} L^{b}\right)-e^{*}-K\right\}+(1-\mu)\left\{v \ln \left(A K^{a}\right)-K\right\},
$$

and

$$
\max _{L} \varphi\left\{w \ln \left(t^{*} A K^{a} L^{b}\right)-f^{*}-L\right\}+(1-\varphi)\{w \ln \bar{c}-L\},
$$

where $\bar{c}$ denotes the outside consumption opportunity endowed upon trespassers when they do not meet with an owner. Note that from the analysis in the previous subsection, $e^{*}, f^{*}, s^{*}$, and $t^{*}$ are all independent of $K$ and $L$. The optimal levels of the inputs for production in the non-cooperative Nash equilibrium can be calculated in a

4 The results in (6) and (7) are special cases of the formulae derived in Eswaran and Neary (2014:220); thus, the proof is omitted. 
straightforward manner, as follows:

$$
K^{*}=a v, L^{*}=\varphi b w
$$

\section{Analysis of the Evolutionary Process}

Here, the preference parameters are chosen by the process of natural selection. This process is governed by the fitness function $\Psi(\mathrm{c}, \mathrm{i}, \mathrm{J})$, defined as

$$
\Psi(\mathrm{c}, \mathrm{i}, \mathrm{J})=\ln (c)-(i+J),
$$

where $c$ denotes consumption, and $i$ and $J$ denote the levels of the distribution effort and production input, respectively. Furthermore, we denote the ratio of the expected consumption by the owner and that by the trespasser as $\Pi$, indicating the level of the property right distributed to the owner relative to that of trespasser.

Using (10) as the subgame perfect level of the production input by the two players, we can write the fitness function (11) for the owner $\sigma(v, w)$ and for the trespasser $\tau(v, w)$ as

$$
\begin{aligned}
& \sigma(v, w)=\mu\left[\ln \left\{s^{*}(v, w) \mathrm{A}(\mathrm{a} v)^{a}(\varphi b w)^{b}\right\}-e^{*}(v, w)-a v\right]+(1- \\
& \mu)\left[\operatorname{lnA}(\mathrm{a} v)^{a}-a v\right] \\
& \quad=\mu g(v, w)+\ln \mathrm{A}(\mathrm{a} v)^{a}+\mu \ln (\varphi b w)^{b}-a v, \\
& \tau(v, w)=\varphi\left[\ln \left\{t^{*}(v, w) \mathrm{A}(\mathrm{a} v)^{a}(\varphi b w)^{b}\right\}-f^{*}(v, w)-\varphi b w\right]+ \\
& (1-\varphi)[\ln \bar{c}-\varphi b w] \\
& \quad=\varphi h(v, w)+(1-\varphi) \ln \bar{c}+\varphi \ln \mathrm{A}(\mathrm{a} v)^{a}+\varphi \ln \mathrm{A}(\varphi b w)^{b}-\varphi b w,
\end{aligned}
$$

where we define

$$
\begin{aligned}
& g(v, w)=\ln \left(s^{*}(v, w)\right)-e^{*}(v, w), h(v, w)=\ln \left(t^{*}(v, w)\right)- \\
& f^{*}(v, w) .
\end{aligned}
$$

The evolution of preferences is characterized by the following two maximization problems:

$$
\max _{v} \sigma(v, w), \max _{w} \tau(v, w) .
$$

The first-order conditions for the two maximization problems are

$$
\begin{aligned}
& \frac{\partial \sigma(v, w)}{\partial v}=\mu \frac{\partial g(v, w)}{\partial v}+a\left(\frac{1}{v}-1\right)=0 \\
& \frac{\partial \tau(v, w)}{\partial w}=\frac{\partial h(v, w)}{\partial w}+b\left(\frac{1}{w}-1\right)=0 .
\end{aligned}
$$

Equations (16) and (17) form the two best-response functions $v^{b r}(w)$ and $w^{b r}(v)$, respectively.

Our principal results are summarized as follows. The proofs of all propositions are given in the Appendix. 


\section{PROPOSITION 1}

(1) The best-response functions $v^{b r}(w)$ and $w^{b r}(v)$ have negative slopes; $v$ and $w$ are strategic substitutes.

(2) The Nash equilibrium ( $\widetilde{v}, \widetilde{w})$ is unique and locally uninvadable.

(3) The evolutionary stable preference parameters $(\tilde{v}, \widetilde{w})$ satisfy the following relationship:

$$
\tilde{v}_{<}^{\geq} \widetilde{w} \text { iff } a_{<}^{\geq} \mu b .
$$

(4) The index of the relative ratio of property rights assigned to the two parties, s, satisfies the relationship

$$
s_{<\frac{1}{2}}^{\geq} \text {iff } a_{<}^{\geq} \mu b .
$$

In our model, the key parameters are $(\tilde{v}, \widetilde{w})$, which reflect the preference intensities of the owner and the trespasser. When one parameter is larger than the other, the person with the larger parameter puts more effort into distribution and more input into production. Considering these effects, the above evolutionary process fixes the relative magnitudes of the two preference parameters. By our assumption on the equality of power for the distribution of the output, this aspect does not influence the difference in the preference parameters. In contrast, if the productivity levels of the players differ, nature has a stronger preference for the consumption of the more productive player, in which case, he is more motivated to produce, as in (18). As a result, the more productive player receives a greater share of the product. Note that the productivity of the owner is measured by the standard productivity parameter $a$ of the Cobb-Douglas production function. However, the substantial productivity of the trespasser is usually measured by the similar parameter $b$, and is discounted by $\mu$. This is because when the ratio of owners to the total population is more than $50 \%$, not all owners can meet with a trespasser. Those owners who do not meet with a trespasser can engage in production independently of the effort of a trespasser. Therefore, the production potential of the trespasser is considered less useful than his technical productivity, from the viewpoint of nature.

To determine the implications of the above results, we focus on the case of constant returns to scale, $a+b=1$. From the definition of $\mu$, we have the following corollary.

\section{Corollary to Proposition 1}

Assume that $\mathrm{a}+\mathrm{b}=1$. The evolutionary stable preference parameters $(\tilde{v}, \widetilde{w})$ and the relative ratio of property rights, s, satisfy the following relationships: 


$$
\begin{aligned}
& \tilde{v}_{<}^{\geq} \widetilde{w} \text { iff } a_{<}^{\geq} \frac{1}{2}\left(\text { if } \theta \leq \frac{1}{2}\right), a_{<}^{\geq} 1-\theta\left(\text { if } \theta>\frac{1}{2}\right) . \\
& \mathrm{s}_{<}^{\geq} \frac{1}{2} \text { iff } a_{<\frac{1}{2}}^{\geq}\left(\text {if } \theta \leq \frac{1}{2}\right), a_{<}^{\geq}-\theta\left(\text { if } \theta>\frac{1}{2}\right) .
\end{aligned}
$$

When the ratio of owners in the population is less than one-half, the intensity of the preferences and the ratio of the right to the property depend only on the relative magnitude of the productivity, $a$, between the two players. However, if the ratio is greater than one-half, they also depend on the fraction of owners within the population, $\theta$. In particular, as $\theta$ approaches one and the fraction of trespassers within the total population become negligibly small, $\tilde{v}>\widetilde{w}$ and $s>\frac{1}{2}$ apply for almost any productivity level of the owner. In this case, the productivity of the trespassers is of negligible importance.

Returning to the general case, we next derive the comparative static results.

\section{PROPOSITION 2}

(1) When the productivity of the owner increases, his preference intensity increases, while that of the trespasser decreases.

(2) When the productivity of the trespasser increases, his preference intensity increases, while that of the owner decreases.

(3) When the density of the population of owners is less than $1 / 2$, the preference intensity parameters of the two players remain unchanged when the density of owners increases. However, when the density of owner is more than $1 / 2$, and then increases, the preference intensity parameter of the owner increases, while that of the trespasser decreases.

\section{Application to Legal Discourse}

In order to exploit the implications of the above propositions, we apply the propositions to the legal reasoning often adopted by the court to resolve disputes. When two people contest a property right in a legal dispute, one method of legal reasoning compares the interests of the two parties in terms of utility, and then divides the right to the property accordingly. ${ }^{5}$ The most difficult problem with this decision is how to

\footnotetext{
5 Note that this method should not be confused with the wealth-maximization criterion for legal judgment adopted by Posner (1986), which is based on the hypothetical compensation principle (or the Kaldor-Hicks criterion, in Posner's notation). While Posner's criterion implies that a legal judgment should be made exclusively based on efficiency, the method explained in the text explicitly introduces distributive equity among the contesting parties by comparing their utility.
} 
determine and compare the intensity of the utility of each party from the property. Even though this is observable information for the conflicting players, it is usually understood as being unverifiable by the court. In usual legal practice, this evaluation is deferred to the deliberation of the court, despite this limitation.

Our propositions in the previous section give important insights in construing the preference intensities of the two disputing players from observable parameters in society. Proposition 1 connects the difference between the preference intensities of the two players to more objective parameters $a, b$, and $\mu$, where $a$ and $b$ represent the respective productivities in developing the land of the two players contesting the rights to the land. From the above proposition, the more productive player can be construed as having a stronger preference for the property. Because $\mu$ denotes the probability of the owner meeting a trespasser, the original owner tends to have a stronger preference for the property. This is true even if his productivity for the land is relatively smaller than that of the trespasser, when the likelihood of the owner meeting a trespasser is sufficiently small. When $\mu$ is small, the investment of the trespasser is less useful, even if his productivity is high, and nature gives the owner a greater chance to develop stronger preference for the property even when his productivity to the land is small.

Information on $\mathrm{a}, \mathrm{b}$ can be collected by the court with the help from the experts on the subject or can be verified to the court through contesting parties by presenting sufficient proof. In contrast, it is more difficult to interpret $\mu$. We understand that it shows if the right to the property is naturally well-determined or ambiguously delineated. In the latter case where $\mu$ is close to unity, therefore, most owners are sued for his right to the property, while in the former case where $\mu$ deviates from unity sufficiently, only small part of the owners is contested their right by the trial in court. As the Proposition 2 states, the more a gets larger than $b$, or $\mu$ gets closer to zero, divergence of the preference intensity by the two players gets larger and hence court can confer more property right on the original owner.

\section{Concluding Remarks}

Our analysis extends the evolutionary analysis of the emergence of property right by Eswaran and Neary (2014) to the case where both natural owner and trespasser have opportunity to improve the value of the property by investing on it using his resource. This change of the natural environment critically make difference to the evolutionary determination of preference intensity parameters by the two players, an owner and a trespasser. Furthermore, our environment can be more naturally applied to the legal reasoning exerted by judges in court. In this sense, we hope that our analysis shed some 
light on the nature of legal reasoning and its associated legal studies by providing with its evolutionary-theoretical foundations. 


\section{REFERENCES}

Besley, T. and M. Ghatak (2010), Property Rights and Economic Development, 4525-4595 in Handbook of Development Economics vol. 5, D. Rodrik and M. Rosenzweig eds., New York: Elsevier.

Coase, R.H. (1960), The Problem of Social Cost, Journal of Law and Economics 3, 1-44.

Cooter, R.C. (1982), The Cost of Coase, Journal of Legal Studies 1, 1-33.

Cressman, R. (2009), Continuously Stable Strategies, Neighborhood Superiority and Two-Player Games with Continuous Strategy Space, International Journal of Game Theory 38, 221-247.

Eswaran, M. and H.M. Neary (2014), An Economic Theory of the Evolutionary

Emergence of Property Rights, American Economic Journal: Microeconomics 6, 203-226.

Hobbes, T. (1651/1973), Leviathan, New York: Collier-MacMillan.

Locke, J. (1689/1988), Two Treatises of Government, ed. by P. Laslett, Cambridge, UK: Cambridge University Press.

Maynard-Smith, J. (1982), Evolution and the Theory of Games, Cambridge, UK: Cambridge University Press.

North, D.C. (1990), Institutions, Institutional Change and Economic Performance, Cambridge, UK: Cambridge University Press.

Posner, R.A. (1986), The Economic Analysis of Law, 3rd ed., Boston: Little, Brown \& Co. 


\section{Appendix}

We first summarize preliminary results on the functions $g(v, w)=\ln \left(s^{*}(v, w)\right)-$ $e^{*}(v, w)$ and $h(v, w)=\ln \left(t^{*}(v, w)\right)-f^{*}(v, w)$, defined in (14). From (6) and (7), we have

$$
\begin{aligned}
& \frac{\partial g}{\partial w}=\frac{\sqrt{w}\left(1-v-e^{*}\right)}{2 v(\sqrt{v}+\sqrt{w})}, \frac{\partial h}{\partial w}=\frac{\sqrt{v}\left(1-w-f^{*}\right)}{2 w(\sqrt{v}+\sqrt{w})}, \\
& \frac{\partial^{2} g}{\partial v \partial w}=\frac{1-v-2 e^{*}}{4 \sqrt{v w}(\sqrt{v}+\sqrt{w})^{2}}, \frac{\partial^{2} h}{\partial v \partial w}=\frac{1-w-2 f^{*}}{4 \sqrt{v w}(\sqrt{v}+\sqrt{w})^{2}} .
\end{aligned}
$$

As pointed out in Eswaran and Neary (2014:222),

$$
\frac{\partial^{2} g}{\partial v^{2}}<0, \text { if } v \leq \frac{5}{3}, \frac{\partial^{2} h}{\partial w^{2}}<0, \text { if } w \leq \frac{5}{3},
$$

and

$$
\Delta=\left(\frac{\partial^{2} g}{\partial v^{2}}\right)\left(\frac{\partial^{2} h}{\partial w^{2}}\right)-\left(\frac{\partial^{2} g}{\partial v \partial w}\right)\left(\frac{\partial^{2} h}{\partial v \partial w}\right)>0, \text { if } v<1 \text { and } w<1 .
$$

\section{Proof of PROPOSITION 1}

Substituting (A.1) into the first-order conditions (16) and (17), we have

$$
\frac{\mu \sqrt{w}}{2 a(\sqrt{v}+\sqrt{w})}=-\frac{1-v}{1-v-e^{*}}>0, \frac{\sqrt{v}}{2 b(\sqrt{v}+\sqrt{w})}=-\frac{1-w}{1-w-f^{*}}>0,
$$

which in turn implies the inequality conditions

$$
1-v>0,1-v-e^{*}<0,1-w>0,1-w-f^{*}<0 .
$$

Therefore, the values $(\tilde{v}, \widetilde{w})$ that satisfy (16) and (17) are both less than unity.

(1) From the inequalities (A.6) and (A.2), $\frac{\partial^{2} g}{\partial v \partial w}<0$ and $\frac{\partial^{2} h}{\partial v \partial w}<0$ at $(v, w)=(\tilde{v}, \widetilde{w})$.

From this result and (A.3), the slopes of the best-response functions are $\frac{\mathrm{d} v^{b r}(w)}{d w}=$ $-\frac{\frac{\partial^{2} \sigma}{\partial v \sigma w}}{\frac{\partial^{2} \sigma}{\partial v^{2}}}<0$ and $\frac{\mathrm{d} w^{b r}(v)}{d v}=-\frac{\frac{\partial^{2} \tau}{\partial v \partial w}}{\frac{\partial^{2} \tau}{\partial w^{2}}}<0$ at $(v, w)=(\tilde{v}, \widetilde{w})$. Therefore, $v$ and $w$ are strategic substitutes at $(v, w)=(\tilde{v}, \widetilde{w})$.

(2) We can readily show from (16), (17), and (A.3) that the second-order conditions for the maximization problem $(15)$ are satisfied for $(\tilde{v}, \widetilde{w})$. Hence, it is a locally unique equilibrium. Then, $(\tilde{v}, \widetilde{w})$ is locally uninvadable because this concept requires only that $(\tilde{v}, \widetilde{w})$ is a neighborhood strict Nash equilibrium, as proved in Cressman (2009).

(3) Using (16) and (17), and substituting in (A.1) we have

$$
\frac{a}{\mu}-b=\frac{1}{2(\sqrt{v}+\sqrt{w})}\left\{\sqrt{v}\left(1-\frac{f^{*}}{1-w}\right)-\sqrt{w}\left(1-\frac{e^{*}}{1-v}\right)\right\} .
$$


Substituting (6) into (A.7) shows that

$$
\frac{a}{\mu}-b=\frac{1}{2(\sqrt{v}+\sqrt{w})}\left(\sqrt{v}-\sqrt{w}+\frac{v w(v-w)}{(1-v)(1-w)(\sqrt{v}+\sqrt{w}}\right)
$$

must be satisfied at $(v, w)=(\widetilde{v}, \widetilde{w})$.

If $\frac{a}{\mu}>b$, suppose that $\tilde{v} \leq \widetilde{w}$. Then, the right-hand side of (A.8) becomes non-positive and the contradiction follows. Therefore, $\tilde{v}>\widetilde{w}$ must be met. Similarly, $\frac{a}{\mu}<b \rightarrow \tilde{v}<\widetilde{w}$. When $\frac{a}{\mu}=b$, suppose $\tilde{v} \neq \widetilde{w}$. Then, the left-hand side of (A.8) is zero and the right-hand side is nonzero, and a contradiction follows. Therefore, $\tilde{v}=\widetilde{w}$ is implied. In summary, we have $\frac{a}{\mu}>b \rightarrow \tilde{v}>\widetilde{w}, \frac{a}{\mu}=b \rightarrow \widetilde{v}=\widetilde{w}$, and $\frac{a}{\mu}<b \rightarrow \tilde{v}<\widetilde{w}$. In proving the opposite implications, we first check directly from (A.8) that $\tilde{v}=\widetilde{w} \rightarrow \frac{a}{\mu}=b$. By taking the contrapositives of $\frac{a}{\mu}>b \rightarrow \tilde{v}>\widetilde{w}$ and $\frac{a}{\mu}<b \rightarrow \tilde{v}<\widetilde{w}$, and using $\tilde{v}=\widetilde{w} \rightarrow \frac{a}{\mu}=b$, we have $\tilde{v}<\widetilde{w} \rightarrow \frac{a}{\mu}<b$ and $\tilde{v}>\widetilde{w} \rightarrow \frac{a}{\mu}>b$. Then, (18) is established by summarizing all the results.

(4) Using the definition of $\mathrm{s}$ in (7) and (18), (19) follows.

\section{Proof of PROPOSITION 2}

Denoting the second-order derivatives of $\sigma$ and $\tau$ around $(\tilde{v}, \widetilde{w})$ as $\sigma_{i j}$ and $\tau_{i j}$, respectively, where $i, j=v, w, a, b, \mu$, the standard comparative static matrix using (16) and (17) is $\left[\begin{array}{cc}\sigma_{v v} & \sigma_{v w} \\ \tau_{w v} & \tau_{w w}\end{array}\right]\left[\begin{array}{l}d v \\ d w\end{array}\right]=\left[\begin{array}{ccc}-\sigma_{v a} d a & 0 & -\sigma_{v \mu} d \mu \\ 0 & -\tau_{w b} d b & 0\end{array}\right]$. Inverting the left-hand side matrix, we have $\left[\begin{array}{l}d v \\ d w\end{array}\right]=\frac{1}{\Delta}\left[\begin{array}{cc}\tau_{w w} & -\sigma_{v w} \\ -\tau_{w v} & \sigma_{v v}\end{array}\right]\left[\begin{array}{ccc}-\sigma_{v a} d a & 0 & -\sigma_{v \mu} d \mu \\ 0 & -\tau_{w b} d b & 0\end{array}\right]$. From (16) and (17), we have $\sigma_{v v}<0$ and $\tau_{w w}<0$, using (A.3). Similarly, from (A.2) and (A.6), $\sigma_{v w}<0$ and $\tau_{w v}<0$. In addition, from (16) and (17), $\sigma_{v a}=\frac{1}{v}-1>0$, $\tau_{w b}=\frac{1}{w}-1>0$, and $\sigma_{v \mu}=\frac{\partial g}{\partial v}<0$.

Using the inverted matrix and the above inequalities, we have 
(A.8)

$$
\frac{\partial v}{\partial a}=-\frac{1}{\Delta} \tau_{w w}{ }_{v a}>0, \frac{\partial w}{\partial a}=\frac{1}{\Delta} \tau_{w v} \sigma_{v a}<0,
$$

(A.9)

$$
\frac{\partial v}{\partial b}=\frac{1}{\Delta} \sigma_{v w} \tau_{w b}<0, \frac{\partial w}{\partial b}=-\frac{1}{\Delta} \sigma_{v v} \tau_{w b}>0,
$$

(A.10)

$$
\frac{\partial v}{\partial \mu}=-\frac{1}{\Delta} \tau_{w w}{ }_{v \mu}>0, \frac{\partial w}{\partial \mu}=\frac{1}{\Delta} \tau_{w v} \sigma_{v \mu}<0 .
$$

From (A.8) and (A.9), results (1) and (2) follow. From $\frac{\partial \mu}{\partial \theta}=0$ if $\theta<\frac{1}{2}$, and $\frac{\partial \mu}{\partial \theta}<0$ if $\theta \geq$ $\frac{1}{2}$, result (3) follows.

\section{Atsushi Tsuneki}

Institute of Social and Economic Research, Osaka University,

6-1 Mihogaoka, Ibaraki, Osaka 567-0047, Japan

tsuneki@iser.osaka-u.ac.jp 